\title{
Bioanalytical method development and validation of biosimilars: lessons learned
}

\begin{abstract}
Biosimilars are expected to be a significant growth driver for the pharmaceutical industry over the next decade, mainly because of the current market penetration of biologics and the need to provide payers cost savings over the originator therapeutics. Legislative support and regulatory guidance have facilitated their entry into pharmacy formularies of the future. Unlike small molecule generic drugs, biosimilars are heterogeneous proteins manufactured using cell-based systems of either microbial or mammalian origin. The use of living systems to manufacture drugs raises challenges in terms of product characterization and therapeutic equivalence to the innovator protein therapeutic. In this article, we share some lessons learned from developing and validating pharmacokinetic and immunogenicity assays that support preclinical and clinical comparative studies for the development of biosimilars.
\end{abstract}

Volume I Issue I - 2014

\author{
Yi Qun Xiao, Andreea Halford, Roger Hayes \\ Hayes
}

Laboratory Sciences Division, MPI Research, USA

Correspondence: Yi Qun Xiao, Director of Immunology, MPI Research, 54943 North Main Street, MI 4907I-9399, Michigan, USA, Tel I-269-668-3336-II89, Fax I-269-668-4I5I, Email YiQunXiao@mpiresearch

Received: April 26, 2014 | Published: May 05, 2014

Keywords: biosimilars, originator, reference product, innovator, ligand binding assay, immunogenicity, interchangeability

\begin{abstract}
Abbreviations: IND, investigational new drug; PK, pharmacokinetics; LBA, ligand binding assays; ECL, electrochemiluminescence; $\mathrm{CRO}$, contract research organization; ADA, anti-drug antibody; LLOQ, lower limit of quantification; MSD, meso scale discovery
\end{abstract}

\section{Introduction}

The global market for biologics has achieved double digit growth in the past 10 years. Meanwhile, it is predicted that nearly $\$ 67$ billion worth of biologic patents will expire by the year 2020. The market growth, cost pressure from governments and insurers, and supporting legislation(s) are the driving forces that make biosimilars the fastest growth sector in the pharmaceutical industry. The US lags behind other regulated markets in terms of biosimilar competition. While the US FDA issued three draft guidance documents on February 9, 2012, to assist the industry in developing biosimilar products in the United States,${ }^{1-3}$ EMA has approved 20 biosimilars within the product classes of human growth hormone, granulocyte colony-stimulating factor, erythropoietin, and TNF-inhibitor. Remsima, a biosimilar to Remicade (infliximab) was approved in South Korea by the biopharmaceutical company Celltrion in July 2012. Following by its partner, Hospira received Inflectra ${ }^{\mathrm{TM}}$ (infliximab) approval in Europe on Sept. 10, 2013 , which became the first biosimilar monoclonal antibody to be approved in Europe. In addition to Remsima, Celltrion also received approval for its cancer treatment biosimilar mAb Herzuma (trastuzumab) in South Korea. Those new biosimilar approvals encourage biopharmaceutical and pharmaceutical industries to actively develop cost saving biologics. Biosimilarity is defined as the high similarity of the biologic product to the reference product, notwithstanding minor differences in clinically inactive components. Moreover, there should be no clinically meaningful differences between the biological product and the reference product in terms of the safety, purity, and potency of the product. ${ }^{1}$ In order to evaluate biosimilarity between an innovator and biosimilar product, the FDA recommended a stepwise, risk-based totality of evidence approach. In addition to extensive structural and functional characterization, effort must also be made on preclinical development in terms of animal toxicity studies and the assessment of pharmacokinetics (PK) and immunogenicity. Although there are FDA, EMA guidelines, and whitepapers describing best practices for bioanalytical method development and validation, ${ }^{4-9}$ there is no clear guidance or recommendation to design and validate bioanalytical assays for biosimilars. The following sections described the experience and lessons we have learned during the course of biosimilar bioanalytical method development and validation.

\section{Choosing the right platform}

In the past four years we have conducted many biosimilar IND enabling drug safety studies with pharmacokinetics and immunogenicity as the critical components, including Rituxan (rituximab, MabThera), Herceptin (trastuzumab), Enbrel (etanercept), Neulasta (pegfilgrastim), Humira (adalimumab) and NESP (darbepoetin alfa). Because these originator drugs were approved some time ago, with the advancement of bioanalytical technologies the PK or immunogenicity methods for biosimilars are not necessarily identical to the ones which were used for the original approval. Ligand binding assays (LBAs) are used to measure the therapeutic level of biologics in biological samples. Many considerations are taken for platform selection. The most commonly used LBA method is ELISA. Compared to electrochemiluminescence (ECL) measurement on Meso Scale Discovery (MSD) and the automated nanoscale immunoassay platform Gyrolab, ELISA's sensitivity and dynamic range are inferior. However, the low cost of an ELISA plate and associated reagent supplies, along with widely used colorimetric plate readers, have rendered a long life for its application. For biologics that require high-doses for efficacy, assay's sensitivity is not normally a determining factor in choosing the detection platform. Oftentimes a Sponsor chooses ELISA simply for ease of transferring the method between their own laboratory and a contract research organization (CRO), or that they simply do not have sophisticated analytical platforms available. On the other hand, both MSD and Gyrolab are more sensitive with broader dynamic range and higher throughput. Most PK assays are developed on MSD in our laboratory when the lower limit of quantification (LLOQ) is required to be at pg/ $\mathrm{mL}$ levels. It is worth noting that matrix effects can be a challenge to resolve in some animal species. In those instances, Gyrolab will be the primary choice because its microfluidic fast ligand interaction minimizes matrix effects. If post-process sample stability has been established the execution of routine sample analysis using the Gyrolab 
workstation becomes a hands-free process. As such, the efficiency and improved data quality will overcome the current high cost of supplies that remains a concern for its wider application.

\section{Assay formats}

It is widely accepted that one PK assay will be used for both biosimilar and originator drugs, ${ }^{10-12}$ therefore, it will not be discussed in any further detail. Depending on the availability of critical reagents, the assay formats are fairly flexible. However, the assay should be developed for its intended purpose. For example, Rituxan can be measured using its target CD20 or anti-idiotype antibody which was not against its CDR for capture.$^{13}$. Due to the presence of soluble CD20 target in circulation, the CD20 PK profile shows significantly lower exposure than an anti-idiotype PK profile.

A common question asked is whether to develop a free assay or a total PK assay? A rule of thumb is that for pharmacodynamic evaluation, what matters most is the amount of free ligand available, whereas for toxicokinetic evaluation, total drug and ligand is preferred. Another biosimilar PK method we have developed using target antigen as the capture reagent is for the measurement of Herceptin, knowing that its target receptor is HER2.

Although a universal PK assay using anti-human IgG antibodies for capture and detection is not a preferred choice in clinical development, it is a reasonable option for preclinical studies especially when the idiotype antibodies are not available. Nonetheless, the most popular PK format for therapeutic antibody biosimilars uses idiotypic antibodies. A successful example in our laboratory was for a Humira biosimilar.

\section{Custom assays versus commercially available kits}

While most biosimilar PK evaluations are conducted using custom-built assays, commercially available kits are used for the PK measurement of certain biosimilars, such as NESP and Neulasta biosimilars. Unlike a custom-built assay where any aspect of the assay procedure can be modified and optimized, a pre-made kit presents the challenge that very few steps within the method may be optimized to ensure successful validation. Regardless of origin (kit or customized assay), the bioanalytical method must meet the validation acceptance criteria outlined within the FDA and EMA guidelines. ${ }^{4,5}$ Most commercial ELISA kits are not intended to be used in the presence of matrix and as such, the success and suitability of using a kit for PK evaluation will depend on its ability to tolerate matrix effects. Additionally, instead of using the recombinant protein calibrator supplied with the commercial kit, the bioanalytical validation must use the biosimilar or innovator therapeutic for the generation of standard curves and QC samples. The selectivity of antibodies used in a commercial kit will have the greatest impact on the ability of the kit to discriminate the analyte of interest at the LLOQ. Most times the plates come pre-coated and the detection antibody is provided at a given concentration and volume. It is therefore important that the kit has an LLOQ that can accommodate the preclinical and clinical study design. The kit must be sufficiently sensitive to detect the biosimilar and originator at the expected circulating levels based on the dosing regimen. Because of the sensitivity requirement and the limitation of critical reagents, occasionally the only feasible option is to use a commercially available kit. As innovator data are readily available, the PK method development and validation should be targeted to the expected exposure data. Such was the case of NESP and its biosimilar within our laboratory. Multiple attempts of using monoclonal and polyclonal antibodies on the ELISA, MSD, and Gyros platforms resulted in a bioanalytical method with $\mathrm{ng} / \mathrm{mL}$ level sensitivity.
However, the route of administration being subcutaneous necessitated that the PK assay had $\mathrm{pg} / \mathrm{mL}$ sensitivity, a 1000-fold difference. The only commercial kit to meet this requirement was the Human Erythropoietin Quantikine IVD ELISA Kit, from R\&D Systems.

As with all critical reagents, when electing to conduct PK evaluation using a commercial kit, inventory and availability must be established up-front. It is not uncommon for ELISA kits to be on backorder which requires lead-time of several weeks, or in some cases, months. This could have detrimental effects on project timelines and regulatory milestones such as the IND submission. If the kit is to be used for clinical PK evaluation, it will be imperative that the necessary kit inventory be secured, and this is best accomplished by maintaining relationships with kit vendors.

\section{One method does not fit all programs}

An interesting phenomenon that we have periodically encountered relates to apparent differences in assay performance for the same therapeutic molecule across multiple programs. A bioanalytical method previously validated for an Enbrel biosimilar program on the MSD platform could not be validated for another Enbrel biosimilar. The bioanalytical method had to be re-developed, optimized, and validated, using a different assay format. How could this happen? We must remember that changes or differences in the manufacturing processes can lead to protein aggregation or post-translational modifications. This may impact the binding characteristics of the molecule to be detected within a given assay format even if those changes do not create any clinically meaningful differences between the biological product and the reference product. Additionally, during the manufacturing and characterization processes, the biosimilar should be compared against a sufficient number of innovator lots so the characterization can confirm that the biosimilar fits within the established ranges for the innovator physicochemical properties.

The above-mentioned example led to an increase in the overall project timeline as well as increased costs for method development. Therefore, it is important that time and financial provisions be made for the inevitable complications that will arise during method development even if the laboratory had previously validated a method for another Sponsor's biosimilar therapeutic.

\section{What does ADA tell you?}

Immunogenicity is evaluated in both preclinical and clinical settings. Although the induction of antibody formation in animals is not indicative of human immunogenicity response, preclinical immunogenicity is typically used to evaluate similarity between the biosimilar and innovator molecule. This is in addition to the extensive battery of physicochemical characterization procedures during the manufacturing process. The immunogenicity incidence for the biosimilar should be equal, or perhaps somewhat less, than that of the innovator. Large differences in immunogenicity incidence can be indicative of conformational differences between the biosimilar and innovator molecules.

As with other biologics in the preclinical setting, immunogenicity evaluation for biosimilar programs is also used to evaluate the impact on the PK profile. In cases where high rates of immunogenicity are observed, such as the case of Enbrel, it was expected and observed that the immunogenicity response has an impact on the PK profile following repeat dose administration. It is therefore important that the immunogenicity assay is able to similarly detect anti-biosimilar and anti-innovator antibodies. If a single immunogenicity assay is used to evaluate immunogenicity of both compounds, then it is advisable that 
the assay be built using the biosimilar molecule so if a bias is created through the use of a single assay, the method will be more sensitive to anti-biosimilar antibodies as opposed to anti-innovator antibodies. If two assays are used, one for each molecule, it is necessary that the two assays have similar sensitivity, specificity, linearity and drug tolerance such that they are similarly impacted by the circulating drugs and can detect similar levels of anti-drug antibodies. ${ }^{11}$ It should be noted that the issue of one assay versus two assays for immunogenicity evaluation is currently a "hot topic" that has not yet achieved consensus between industry and regulators. ${ }^{10-15}$

While PK assays follow multiple formats, the standard immunogenicity assay relies on a bridging format wherein the drug is used for both capture and detection of the ADA. The advantage of the bridging format lies in the fact that it only uses the variable regions of the ADA positive control, thus allowing the use of surrogate positive controls without the need for matching the species in which the immunogenicity response is to be measured. The MSD platform has become the gold standard for bridging immunogenicity assays. The platform has the added advantage of facilitating a homogeneous assay, eliminating washing steps, and allowing for the detection of low affinity responses. However, the ability to develop a bridging immunogenicity assay is dependent on the type of biosimilar or innovator therapeutic. For example, while immunogenicity assays could be developed using the bridging format for biosimilars to Enbrel, Herceptin, and Rituxan, this could not be done for NESP and its biosimilar. This is likely due to the relatively small size of the molecule as well as its heavy glycosylation. Immunogenicity evaluation for the NESP biosimilar program was conducted using a direct ELISA immunogenicity assay format, wherein the biotinylated NESP innovator or biosimilar were coated onto the streptavidin plate as capture reagents while anti-species Fc antibody against the isotype of the surrogate positive control was used for detection. The disadvantage of such a method is that if the positive control is not generated from the same species as the samples that will be analyzed, additional controls must be incorporated into assay development and validation to confirm that the assay format can actually detect antidrug antibodies generated by dosed animals.

Ultimately, regardless of bridging or direct ELISA, all immunogenicity formats are subject to interference from circulating drugs. The best way to overcome drug interference is to design the preclinical and clinical studies to ensure the immunogenicity samples are collected at intervals with low levels of circulating drugs. This would require longer recovery periods for preclinical studies and long follow up visits for clinical programs. An alternate way of overcoming the drug interference problem and increasing the drug tolerance of the assay is incorporating an acid dissociation treatment of the samples before analysis. While acid dissociation increases the drug tolerance level of an assay, it may potentially damage the drug-antibody binding interactions, thus potentially altering the final immunogenicity results. The Gyrolab platform offers an elegant solution for the acid dissociation step. Through the use of a special disk equipped with a mixing chamber, the Gyrolab platform has introduced a controlled environment for acid dissociation and subsequent neutralization which is fully automated and occurs within a time frame of a few seconds.

\section{Questions still need to be answered}

Although there is a global agreement for the need and potential of biosimilar therapeutics, many issues regarding clinical development remain. For instance, although the US regulatory guidelines refer to biosimilars, the terminology varies widely across the globe.
Biosimilars are referenced as follows: 'similar biological medicinal product' in EU; 'similar biotherapeutic product (SBP)' by the WHO; 'biologic product' in Brazil; 'similar biologic' in India; 'biocomparable' in Mexico; and 'subsequent-entry biological' in Canada, to name a few. In addition to the differences in terminology, there are points of agreement and points of difference between the EMA and FDA regulatory guidelines pertaining to biosimilar development. Both agencies agree on the inherent complexity of the molecules and the production process. They also recommend a step-wise approach to drug development rooted in a 'totality of evidence' requiring $\mathrm{CMC}$, preclinical as well as clinical data to confer biosimilarity. Both agencies recognize the need to ensure global drug supply and are therefore accepting the use of non-EEA or non-US licensed innovator product, provided that the appropriate bridging studies are conducted. This agreement was recently reached, as both EMA and US FDA guidelines currently state comparator product must be the product marketed in the territory in question. However, the EU and US markets are often supplied by different manufacturing sites for a given product. Even when all products are made in one plant, final filling for EU and US markets may be conducted at different sites and in different containers/closures. Therefore, the FDA has indicated that if analytical, nonclinical, and pharmacokinetic equivalence is demonstrated between US and EU marketed products, then they would allow an EU marketed product to be used as a comparator in Phase III trials for US approvals. EU regulators have also accepted the approach outlined above by US FDA for Phase III trials. Finally, both agencies require post-marketing safety monitoring and immunogenicity evaluation pre- and post-approval.

However, along with the above items of agreement, the two agencies have several areas of disagreement. While EMA considers biosimilarity sufficient for approval, leaving the issue of interchangeability (whether required or not) to be addressed by the individual member states, the FDA requires both biosimilarity and the claim of interchangeability with the reference product before approval is granted. Moreover, the US reference (not the EEA-licensed) is required for the conduct of interchangeability studies. The agencies also take a divided stance on the need for preclinical studies. EMA does not recommend preclinical studies for investigating general toxicity in non-relevant species, while the FDA requires preclinical studies for comparison of general toxicity, especially if different expression systems were used for manufacture. Finally, a current hot topic within the biosimilar field revolves around the importance of naming, more precisely the International Non-proprietary Names (INN) such as infliximab or rituximab. On one hand, biosimilar companies are attempting to make a case for approval of their molecule under the same INN as the innovator. This is met with opposition by not only innovator companies but also by physicians who are uncomfortable with the idea of a single INN and fear that this will impair tracking and reporting of adverse effects. The end result of this ongoing battle can have substantial effects on biosimilar market entry and uptake. ${ }^{16-22}$

\section{Conclusion}

The current review is intended to provide some insight of bioanalytical challenges to support biosimilars. With significantly increased effort on biosimilar development, the pharmaceutical industry and regulated authorities will gain further experience for the best-practices, strategies, and global harmonization in this fast growing area.

\section{Acknowledgements}

None. 


\section{Conflicts of interest}

Author declares there are no conflicts of interest.

\section{Funding}

None.

\section{References}

1. FDA Quality Consideration in Demonstrating Biosimilarity to a Reference Protein Product.

2. FDA Guidance for Industry: Scientific considerations in demonstrating biosimilarity to a reference product.

3. FDA Biosimilars: Questions and Answers Regarding Implementation of the Biologics Price Competition and Innovation Act of 2009.

4. US Department of Health and Human Services. US FDA, Center for Drug Evaluation and Research. Guidance for industry:bioanalytical method validation. MD, USA: Rockville; 20101.

5. European Medicines Agency.Guideline on bioanalytical method validation. London, UK: Committee for Medicinal Products for Human use (CHMP); 2011.

6. DeSilva B, Smith W, Weiner R, et al. Recommendations for the bioanalytical method validation of ligand-binding assays to support pharmacokinetic assessments of macromolecules. Pharm Res. 2003;20(11):1885-1900.

7. Kelley M, DeSilva B. Key elements of bioanalytical method validation for macromolecules. AAPS J. 2007;9(2):E156-E163.

8. Koren E, Smith HW, Shores E, et al. Recommendations on risk-based strategies for detection and characterization of antibodies against biotechnology products. J Immunol Methods. 2008;333(1-2):1-9.

9. Shankar G, Devanarayan V, Amaravadi L, et al. Recommendations for the validation of immunoassays used for detection of host antibodies against biotechnology products. J Pharm Biomed Anal. 2008;48(5):1267-1281.

10. Cai XY, Gouty D, Baughman S, et al. Recommendations and requirement for the design of bioanalytical testing used in comparability studies for biosimilar drug development. Bioanalysis. 2011;3(5):535-540.
11. Islam R. Bioanalytical challenges of biosimilars. Bioanalysis. 2014;6(3):349-356.

12. Oldfield P. Differences in bioanalytical method validation for biologically derived macromolecules (biosimilars) compared with small molecules (generics). Bioanalysis. 2011;3(14):1551-1553.

13. Kuang B, King L, Wang HF. Therapeutic monoclonal antibody concentration monitoring:free or total? Bioanalysis. 2010;2(6):11251140 .

14. Cai XY, Thomas J, Cullen C, et al. Challenges of developing and validating immunogenicity assays to support comparability studies for biosimilar drug development. Bioanalysis. 2012;4(17):2169-2177.

15. Nicholson R, Lowes S, Caturla MC, et al. Conference report: 6th GCC focus on LBA: critical reagents, positive controls and reference standards; specificity for endogenous compounds; biomarkers; biosimilars. Bioanalysis. 2012;4(19):2335-2342.

16. European Medicines Agency Guideline on Similar Biological Medicinal Products containing monoclonal antibodies - non-clinical and clinical issues.

17. European Medicines Agency Guideline on Similar Biological Medicinal Products containing biotechnology - derived proteins as active substance: non-clinical and clinical issues.

18. European Medicines Agency Guideline on similar biological medicinal products containing monoclonal antibodies-non-clinical and clinica issues.

19. Biologics Price Competition and Innovation Act (BPCIA) of 2009 interchangeability reference.

20. World Health Organization Guidelines on evaluation of similar biotherapeutic products (SBPs).

21. Guidelines on similar biologics: Regulatory Requirements for Marketing Authorization in India.

22. Health Canada Guidance for Sponsors: Information and Submission Requirements for Subsequent Entry Biologics (SEBs). 